\title{
Clinically feasible reconstruction of 3D whole-body PET/CT data using blurred anatomical labels
}

\author{
Claude Comtat ${ }^{1}$, Paul E Kinahann ${ }^{2,3}$, Jeffrey A Fessler ${ }^{4}$, Thomas Beyer ${ }^{5}$, \\ David W Townsend ${ }^{3}$, Michel Defrise ${ }^{6}$ and Christian Michel ${ }^{5}$ \\ ${ }^{1}$ Service Hospitalier Frédéric Joliot, Orsay, France \\ 2 Department of Radiology, University of Pittsburgh, Pittsburgh, PA, USA \\ ${ }^{3}$ Department of Radiology, University of Washington, Seattle, WA, USA \\ ${ }^{4}$ Department of Electrical Engineering and Computer Science, University of Michigan, \\ Ann Arbor, MI, USA \\ ${ }^{5}$ CTI PET Systems Inc., Knoxville, TN, USA \\ ${ }^{6}$ Division of Nuclear Medicine, Vrije Universiteit Brussel, AZ-VUB, Brussels, Belgium \\ E-mail: kinahan@u.washington.edu
}

Received 5 March 2001, in final form 10 September 2001

Published 29 November 2001

Online at stacks.iop.org/PMB/47/1

\begin{abstract}
We present the results of utilizing aligned anatomical information from CT images to locally adjust image smoothness during the reconstruction of threedimensional (3D) whole-body positron emission tomography (PET) data. The ability of whole-body PET imaging to detect malignant neoplasms is becoming widely recognized. Potentially useful, however, is the role of whole-body PET in quantitative estimation of tracer uptake. The utility of PET in oncology is often limited by the high level of statistical noise in the images. Reduction in noise can be obtained by incorporating a priori image smoothness information from correlated anatomical information during the reconstruction of PET data. A combined PET/CT scanner allows the acquisition of accurately aligned PET and x-ray CT whole-body data. We use the Fourier rebinning algorithm (FORE) to accurately convert the 3D PET data to two-dimensional (2D) data to accelerate the image reconstruction process. The $2 \mathrm{D}$ datasets are reconstructed with successive over-relaxation of a penalized weighted least squares (PWLS) objective function to model the statistics of the acquisition, data corrections, and rebinning. A 3D voxel label model is presented that incorporates the anatomical information via the penalty weights of the PWLS objective function. This combination of FORE + PWLS + labels was developed as it allows for both reconstruction of $3 \mathrm{D}$ whole-body data sets in clinically feasible times and also the inclusion of anatomical information in such a way that convergence can be guaranteed. Since mismatches between anatomical (CT) and functional (PET) data are unavoidable in practice, the labels are 'blurred' to reflect the uncertainty associated with the anatomical information. Simulated and experimental results show the potential advantage of incorporating anatomical information by using blurred labels to calculate the penalty weights. We conclude that while the
\end{abstract}


effect of this method on detection tasks is complicated and unclear, there is an improvement on the estimation task.

\section{Introduction}

The role of whole-body PET imaging with [18F] fluorodeoxyglucose (FDG) in oncology research and patient care is increasing (Rigo et al 1996, Weber et al 1999), and its ability to detect malignant neoplasms is becoming widely recognized. Potentially useful, however, is the role of whole-body PET in quantitative estimation of tracer uptake for the purposes of patient management, including staging, monitoring for the effect of therapeutic interventions and/or recurrence and for therapy planning.

The utility of whole-body FDG PET scanning, however, is often limited by the high level of statistical noise in the images. Incorporating a model of the data acquisition statistics can reduce noise propagation in the reconstructed image, as discussed in the reviews by Ollinger and Fessler (1997) and Leahy and Qi (2000). In addition, several studies have shown advantages of using aligned anatomical information to guide the reconstruction of PET data (Fessler et al 1992, Gindi et al 1993, Zhou et al 1993, Ouyang et al 1994, Ardekani et al 1996, Bowsher et al 1996). In other words, reduction in noise can be obtained by incorporating a priori image smoothness information from correlated anatomical information during the reconstruction of the PET data. For thorax or abdomen imaging, however, it is difficult to accurately align anatomical information with PET data. The combined three-dimensional (3D) PET/CT scanner (Beyer et al 2000) resolves the alignment problem by acquiring both functional (PET) and anatomical (CT) data in a single patient scan. The primary goal of acquiring accurately aligned PET and $\mathrm{CT}$ data is to provide accurate anatomical localization of the functional data, for example, determining the precise location of a focal point of FDG accumulation indicative of a malignant neoplasm. The CT data can be acquired as a rapid post-injection transmission scan and used to correct the PET data for attenuation (Kinahan et al 1998), which makes the PET/CT scanner ideal for the estimation of tracer uptake concentration. An additional synergistic combination of the PET and CT data includes using the CT data for guiding the reconstruction of whole-body PET data, with the goal of reducing statistical noise, improving quantitation and potentially improving lesion detectability.

The method we currently use clinically (without including anatomical information) to reduce the effect of statistical noise in the PET images is a combination of Fourier rebinning (FORE) (Defrise et al 1997) followed by reconstruction with the accelerated two-dimensional (2D) ordered-subsets EM reconstruction algorithm (OSEM) developed by Hudson and Larkin (1994), or the attenuation weighted OSEM algorithm (AWOSEM) (Comtat et al 1998).

In these methods 3D PET data are accurately rebinned to a set of contiguous 2D sinograms by applying the Fourier rebinning technique (FORE) prior to the iterative reconstruction. This approach allows reconstruction of 3D PET data in clinically feasible computation times (Kinahan et al 1997) as the use of rebinning methods significantly accelerate image reconstruction since the back- and forward-projection steps are much less computationally demanding in 2D than in 3D. The FORE algorithm causes a small spatially varying distortion of the reconstructed point spread function (PSF) (Defrise et al 1997). In whole-body PET imaging with current scanner geometries, however, the effective image resolution is dominated by the count-limited statistics, and there is no significant difference in image SNR between FORE + AWOSEM and the more accurate fully-3D AWOSEM (Liu et al 2001). 
The aim of this paper is to investigate the potential gains in image signal-to-noise ratio by incorporating the anatomical information derived from aligned CT images in the reconstruction of 3D whole-body PET data, with the constraint that the technique is routinely useable in a clinical environment. An important consideration is to retain the fast reconstruction times of the FORE + OSEM approach. To this end, we combine FORE-rebinning with minimizing a penalized weighted least-squares (PWLS) cost function. The PWLS algorithm is used, rather than OSEM or AWOSEM, as it is straightforward to include (i) anatomical information in such a way that rapid convergence can be guaranteed and (ii) the 3D nature of the regularizing prior, as described below. The 'weighting' in the PWLS includes the statistics of the data acquisition and processing and the FORE algorithm (Comtat et al 1998). The PWLS objective function used in 2D image reconstruction (Sauer and Bouman 1993, Fessler 1994) is given by

$$
\Phi(\boldsymbol{x})=\sum_{j}\left(\sum_{i} A_{j i} x_{i}-y_{j}\right)^{2} \sigma_{j}^{-2}+\beta U(\boldsymbol{x} ; \boldsymbol{l}) .
$$

In equation (1), $\boldsymbol{x}=\left\{x_{i} \mid i=1, \ldots, n\right\}$ is a vector of the $n$ voxel values of the image, $\boldsymbol{y}=\left\{y_{j} \mid j=1, \ldots, m\right\}$ are the $m$ projection values, $\sigma=\left\{\sigma_{j} \mid j=1, \ldots, m\right\}$ are the known (or estimated) standard deviations for the projection data, $\boldsymbol{A}=\left\{A_{j i}\right\}$ is the $m \times n$ system matrix such that the expectation value for $y_{j}$ is given by $\sum_{i} A_{j i} x_{i}$. The parameter $\beta$ controls the influence of the quadratic image roughness penalty function, $U(\boldsymbol{x} ; \boldsymbol{l})$, which we modify to incorporate the 3D anatomical information, termed labels, $l=\left\{l_{i} \mid i=1, \ldots, n\right\}$, as described in the next section. The images are reconstructed by minimizing $\Phi(\boldsymbol{x})$ while keeping $\boldsymbol{l}$ constant. As $\Phi(\boldsymbol{x})$ is a quadratic function it can be readily minimized by the successive over-relaxation (SOR) algorithm in 10-20 iterations (Sauer and Bouman 1993, Fessler 1994). The PWLS method is based on the assumption that the mean and variance of the sinogram data are known after the necessary quantitative corrections are applied for effects such as detector efficiency variations, attenuation, and random and scattered coincidences. If the data are Gaussian distributed, then the PWLS method converges to a maximum-likelihood estimator. This avoids the complexity of accurately propagating the Poisson distribution of the raw sinogram data through a data acquisition model, but requires a separate estimate of the data variance.

In our implementation the 3D PET data are first rebinned with FORE to a set of transaxial $2 \mathrm{D}$ sinograms. The effects on the sinogram data variance of all correction steps, including corrections for attenuation, scattered and random coincidences, detector efficiencies and the effect of FORE, are included in $\sigma^{2}$ (Comtat et al 1998). To retain the 3D nature of the patient tracer distribution, the regularizing term $U(\boldsymbol{x} ; \boldsymbol{l})$ is estimated using a 26-voxel threedimensional local neighbourhood of each voxel, as proposed by Mumcuoglu et al (1994).

An important aspect of any reconstruction method that incorporates anatomical a priori information is the effect of positional and/or signal mismatch between the anatomical and functional data. We expect some level of positional mismatch between the PET and CT images due to, for example, respiration or patient movement during the scan. By signal mismatch we mean that changes in the PET emission distribution are not matched with corresponding changes in the anatomical image, or vice versa. It is important to emphasize that positional and signal mismatches are fundamentally different effects, as we have shown below.

There are two types of signal mismatch. The first is where a change in the PET emission distribution is not matched by a corresponding change in the anatomical image. In other words, there is no change in the labelling or classification of voxels that cross the change in emission distribution, which corresponds to regularization without using anatomical information. This is termed a missing label. The second type of signal mismatch is the opposite situation where a truly uniform emission region has two or more different label sub-regions. The introduction 
of such false labels can lead to dramatic changes in the noise texture, as illustrated below. Both types of signal mismatch are known to occur in practice, due to, for example, the heterogeneous uptake of PET tracers within a single anatomical structure such as a tumour (Rigo et al 1996).

Any improvement in task performance would necessarily depend on the type of study, such as tracer uptake quantitation versus tumour detection. Estimating the effect on detectability is complicated by the availability of the aligned CT image. In this paper we do not try to answer these questions, but rather focus on the estimation task by using simulations to investigate changes in root mean square (RMS) errors and contrast/noise trade-offs by incorporating $\mathrm{CT}$ anatomical information into the PET reconstruction. This is done by modifying the regularizing term, $U(\boldsymbol{x} ; \boldsymbol{l})$, and comparing the results to the standard PWLS method (Sauer and Bouman 1993, Fessler 1994, Comtat et al 1998). Simulation studies allow the mean and standard deviation images to be estimated from multiple independent realizations. The effects of both positional and signal mismatches between the PET and CT data, which are unavoidable in practice, are also evaluated. We also evaluate a 'blurred label' method (described in the next section) of including the CT data in $U(\boldsymbol{x} ; \boldsymbol{l})$ that attempts to reduce the deleterious effects of mismatches between the PET and CT data. Finally, we demonstrate the efficacy of the blurred labels method with experimental phantom data obtained from the PET/CT scanner.

\section{Inclusion of anatomical information}

To include anatomical information we modify the regularizing term, $U(\boldsymbol{x} ; \boldsymbol{l})$, which is a quadratic roughness penalty based on a 3D voxel neighbourhood $\left(\mathrm{N}_{3 \mathrm{D}}\right)$ consisting of a voxel's 26 closest neighbours. The penalty is defined by:

$$
U(\boldsymbol{x} ; \boldsymbol{l})=\frac{1}{2} \sum_{i} \sum_{k \in N_{3 \mathrm{D}}(i)} \frac{\omega_{i k}(\boldsymbol{l})}{d_{i k}}\left(x_{i}-x_{k}\right)^{2}
$$

where $d_{i k}$ is the Euclidean distance between voxels $i$ and $k$, and the penalty weights, $\omega_{i k}(\boldsymbol{l})$, are derived from the anatomical data by using voxel labels, $l$. Voxel labels have previously been used to incorporate anatomical information into emission tomography reconstruction (Johnson et al 1995). In this approach two quantities are associated with an image voxel: the estimated emission density and the class of material to which it belongs (e.g. lung, bone, or soft tissue). In the present paper we define binary penalty weights with $\omega_{i k}$ set to one if both voxels $i$ and $k$ belong to the same class and to zero otherwise. We refer to this as the binary label method. In other words, at the boundary between voxels with different labels it is assumed that there is a potential change in the underlying tracer concentration. In this case no smoothing is applied between these voxels, allowing preservation of the edge in the tracer concentration image. With the use of an edge-preserving penalty function (equation (2)), the smoothing parameter, $\beta$ (equation (1)), can be increased to reduce statistical noise in areas that are assumed to be smoothly varying or uniform, without any blurring of the edges.

The use of binary penalty weights, however, may introduce unacceptable artefacts if there are mismatches between the anatomical and functional images. Alternative methods of including anatomical prior information include the joint estimation approach used with the 'line-site' method proposed by Geman and Geman (1984), where the line-sites reflect the boundaries between voxels in the emission image. The use of anatomical a priori information, such as CT or MRI images, to 'guide' the positions of emission boundaries, estimated jointly with the emission distribution, has been investigated by Leahy and Yan (1991), Gindi et al (1993), and Bowsher et al (1996) and others. Joint estimation can thus potentially compensate for alignment errors (Zhou et al 1993), as the fixed anatomical a priori information is not used to force the position of functional boundaries, but rather is combined with the PET 
emission data to determine the boundary locations. Joint estimation of the anatomical and functional data has also been proposed (Hurn et al 1996). These methods are computationally intensive in the 2D image reconstruction and are currently not suitable for routine clinical processing, particularly for 3D whole-body PET imaging. In addition, the use of a full set of $3 \mathrm{D}$ regular and diagonal 'plane' sites between voxels for the 3D penalty term, $U(\boldsymbol{x} ; \boldsymbol{l})$, would lead to complex book-keeping. Rather than compensating for positional or signal mismatch, a simpler (non-Bayesian) but less precise alternative consists of degrading the resolution of the anatomical information before incorporation into the PET image reconstruction as proposed by Fessler et al (1992) in his 2D blurred weight method. This has the effect of reducing the deleterious effects of anatomical/functional mismatches, but at the same time limits the maximum achievable accuracy (e.g. resolution recovery) that can be obtained by using the anatomical information. In contrast, Bayesian approaches can potentially utilize the full accuracy of the anatomical information while also circumventing the problems introduced by anatomical and functional mismatches (as demonstrated by Zhou et al 1993), albeit at the cost of increased computation time.

In the blurred weight method the binary penalty weights used for reconstructing an image plane are smoothed with a 2D kernel whose width corresponds to the uncertainty in the anatomical information. We have developed and implemented a 3D extension of the blurred weight method that we refer to as the 3D blurred label method that is useable with any 3D topology. In our implementation, rather than smoothing the binary weights $\omega_{i k}$, we introduce an intermediate representation of 3D object labels, which are smoothed. The penalty weights in equation 2 are then derived from the blurred labels, as described next.

In the blurred label method, 3D label maps $l_{c}=\left\{l_{i c} \mid i=1, \ldots, n ; 0 \leqslant l_{i c} \leqslant 1\right\}$ are defined for each class (or label) $c$ of the $C$ classes of material. An example with $C=5$ could be $c=1$ for voxels in the lung region, $c=2$ for soft tissue, $c=3$ for bone, $c=4$ for outside the body and $c=5$ for voxels of unknown type. In this case $l_{1}=\left\{l_{i 1} \mid i=1, \ldots, n ; 0 \leqslant l_{i 1} \leqslant 1\right\}$ is an image or support map of the lung, that is $l_{i 1}=1$ if voxel $i$ is considered to be in the lung, and is $l_{i 1}=0$ if not. The label maps are initialized with mutually exclusive binary values (only one label is associated with each voxel) according to the CT image segmented into the $C$ classes. Each label map is then blurred with the same 3D Gaussian kernel whose width corresponds to the PET-CT alignment accuracy. Thus, before and after smoothing the labels satisfy the relationship $\sum_{c=1, C} l_{i c}=1$, for every voxel $i$. The labels, with or without smoothing, are used to define the penalty weights between voxels as

$$
\omega_{i k}=\sum_{c=1}^{C} l_{i k} l_{k c}
$$

which have the desirable properties that $0 \leqslant \omega_{i k} \leqslant 1$ and $\omega_{i k}=\omega_{k i}$. If $\omega_{i k}=1$ (from the anatomical data) then voxels $i$ and $k$ are assumed to be related functionally in some sense and the full amount of smoothing, as determined by $\beta$ in equation (1), is applied. If $\omega_{i k}<1$ then voxels $i$ and $k$ are considered less likely to be related functionally and a reduced level of local smoothing is imposed. If $\omega_{i k}=0$, no roughness penalty is imposed between voxels $i$ and $k$. Note that in the case of unsmoothed labels, the weights are binary, with $\omega_{i k}=0$ or 1 , which we define as the binary labels method. For the case of no labels, $\omega_{i k}=1$ for all voxels $i$ and $k$, and we return to the PWLS algorithm.

Initial anatomical classification of the voxels can be obtained by segmenting the CT image with thresholding or statistical methods, such as described by Karssemeijer (1990). For the studies reported here, the voxel labeling was accomplished with simple thresholding. The CT image planes are $512 \times 512$, compared to $128 \times 128$ for PET, for the same field of view of $\sim 50 \mathrm{~cm}$. To convert the anatomical information to the larger PET voxel size, we set the label of the PET voxel to that of the majority of the CT sub-voxels. Other initialization methods 


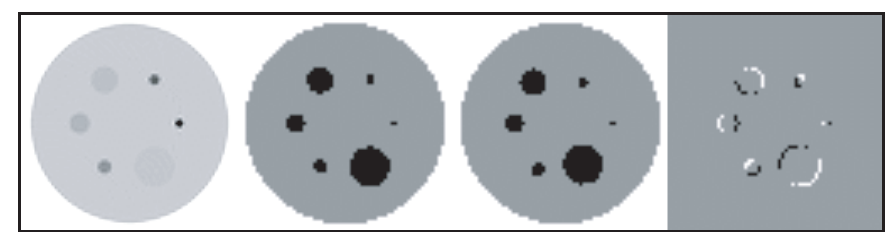

(a)

(b)

(c)

(d)

Figure 1. Emission distribution and label maps used for simulation studies. From left to right: (a) emission distribution (contrast varies with diameter), (b) original label map, (c) shifted label map, and (d) subtraction of original and shifted labels showing the different directions of the label shifts.

can be envisaged, however, such as setting $l_{i c}$ to the fraction of the sub-voxels belonging to tissue class $c$. We note that since $\omega_{i k}$ are fixed, $\Phi(\boldsymbol{x})$ (equation (1)) is still a convex quadratic objective function and so convergence to a unique minimum solution is guaranteed.

\section{Methods}

\subsection{Simulation studies}

The simulation studies use a technique that allows us to generate multiple realizations of 3D sinogram datasets in a feasible computing time (Rowe and Dai 1992, Furuie et al 1994, Comtat et al 1999). We do not simulate the detection of individual true, random and scattered coincidences, as is done with Monte Carlo methods, but rather simulate their effect on the noise of the emission data. We assume that the measured data are accurately corrected for attenuation and random and scattered coincidences. With the exception of the noiseless 2D simulation, all simulations with statistical noise were performed in 3D and reconstructed with FORE + PWLS. For each of these volume images, only the central transverse image plane is displayed. In all simulation studies two label classes were used: one for the cylinder background and one for the embedded discs.

3.1.1. Noiseless $2 D$ data. We first investigated the effect of accurate and mismatched labels in a noiseless $128 \times 128$ ( $2 \mathrm{D}$ only) simulation. The simulation was of a large uniform disc (30-pixel diameter) with a smaller disc (2-pixel diameter) in the centre. The 2D image was reconstructed with a fixed $\beta$ and the following variations of the labels and penalty weights: (i) no labels, (ii) correct binary labels, (iii) correct blurred labels, (iv) shifted binary labels, and (v) shifted blurred labels.

3.1.2. Noisy 3D data. A large uniform cylinder (30 $\mathrm{cm}$ diameter) with six embedded smaller discs of different sizes and contrast levels was the basis for 100 realizations with added statistical noise. Figure 1 shows the original object and the corresponding labels.

The size of the contrast discs ranged from 1 to $6 \mathrm{~cm}$ in diameter and were chosen such that the disc area approximately doubled for each increase in diameter. The contrast levels of the discs varied inversely with area, as proposed by Furuie et al (1994), with the smallest disc having the maximum contrast level of 2.25 relative to the uniform disc. The inverse variation of contrast with area results in the integrated contrast for each object being the same.

The statistical noise level was set to correspond to a total of $2 \times 10^{7}$ coincident events $\left(5.5 \times 10^{6}\right.$ true coincidences, $4.5 \times 10^{6}$ scattered coincidences and $10 \times 10^{6}$ random coincidences), typical of the detected counts (before data corrections) in a 10 min clinical scan 
with the PET/CT scanner. The effects of random and scattered coincidences and attenuation correction were included, with the attenuation coefficient of all objects set to that of water.

Simulation studies were performed for three conditions:

1. with accurate alignment;

2. with the labels of the six contrast discs shifted, in different directions, by $5 \mathrm{~mm}$ relative to the true source distribution using the displacements shown in figure 1(d);

3. with the emission source distribution set to a uniform activity distribution, but with the (un-shifted) labels still included as 'false' labels.

For each of the three-label variations (accurate, shifted and false), the 100 realizations were reconstructed with five different types of penalty weights:

1. no labels $\left(\omega_{i k}=1\right)$;

2. binary labels $\left(\omega_{i k}=0\right.$ or 1$)$;

$3-5$. blurred labels $\left(0 \leqslant \omega_{i k} \leqslant 1\right)$, smoothed with a Gaussian kernel of FWHM 4.0, 5.0 and $7.5 \mathrm{~mm}$.

The images were reconstructed with a set of image smoothness parameters ( $\beta$ in equation (2)) that, when used with PWLS, roughly matched the range of cut-off frequencies used with standard filtered-backprojection in clinical imaging (Comtat et al. 1998).

For each of the combinations of reconstruction parameters, voxel-wise mean and standard deviation images were calculated from the $N=100$ realizations. Additionally, 2D region of interest (ROI) figures of merit were calculated for each of the $i=1, \ldots, 6$ objects: the mean estimated bias, the standard deviation and the RMS error.

Mean estimated bias: $b_{i}$, of object $i$, is given by

$$
b_{i}=\frac{C_{i}^{T}-\bar{C}_{i}}{C_{i}^{T}}
$$

where $C_{i}^{T}$ is the true contrast for object $i$. The mean contrast for each object is given by

$$
\bar{C}_{i}=\frac{1}{N} \sum_{j=1}^{N} C_{i j}
$$

The contrast for each realization of each object is given by

$$
C_{i j}=\bar{R}_{i j}-B
$$

where $B$ is the true background activity and $\bar{R}_{i j}$ is the mean ROI value of object $i$ for realization $j, j=1, \ldots, N$.

Standard deviation: $s_{i}$, of object $i$, is given by

$$
s_{i}=\sqrt{\frac{\sigma_{i}^{2}+\sigma_{B_{i}}^{2}}{2}}
$$

where the object and background variances are given by

$$
\sigma_{i}^{2}=\frac{1}{N-1} \sum_{j=1}^{N}\left(\bar{C}_{i}-C_{i j}\right)^{2} \quad \sigma_{B_{i}}^{2}=\frac{1}{N-1} \sum_{j=1}^{N}\left(\bar{R}_{B_{i}}-\bar{R}_{B_{i} j}\right)^{2}
$$

with mean background

$$
\bar{R}_{B_{i}}=\frac{1}{N} \sum_{j=1}^{N} \bar{R}_{B_{i} j} \approx B
$$

Here, $\bar{R}_{B_{i} j}$ is the mean ROI value in a background region of the same size as object $i$. The standard deviation figure of merit was chosen to include the effect of background noise, which 
can be substantially affected by the use of anatomical a priori information, as changes in the background noise can influence detection tasks.

The location and size of the ROIs for the mean estimated bias and the standard deviation coincide with the true object and their locations were the same for all three cases of accurate labels, shifted labels and false labels. The mean estimated bias and standard deviation were plotted for each object as a function of the smoothness parameter, $\beta$, and for each of the five types of penalty weights listed above.

$R M S$ error: $\mathrm{RMS}_{i}$, of object $i$, is given by

$$
\mathrm{RMS}_{i}=\sqrt{\frac{1}{N} \frac{1}{n_{i}} \sum_{j=1}^{N} \sum_{k=1}^{n_{i}}\left(x_{j, k}-t_{k}\right)^{2}}
$$

where $x_{j, k}$ is the value of voxel $k$ for realization $j$ and $t_{k}$ is the true voxel value. Because the use of labels modifies the voxel values not only within, but also around the object $i$, the summation of the squared difference $\left(x_{j, k}-t_{k}\right)^{2}$ was taken over the voxels $k$ within a circular ROI centered on the true location of object $i$ and with a radius $1 \mathrm{~cm}$ larger than the object radius. The RMS errors were plotted as a function of the smoothness parameter, $\beta$, for four types of penalty weights (no labels, binary labels and labels blurred with a kernel FWHM of 5 and $7.5 \mathrm{~mm}$ ).

\subsection{Experimental studies}

To evaluate the efficacy of the blurred label method in practice, we acquired CT and aqueous [18F] PET scans of a $30 \mathrm{~cm}$ (major axis) elliptical torso phantom in the PET/CT scanner. The phantom had a series of hot and cold contrast spheres, each of which also contained a dilute iodine-based CT contrast agent to aid the segmentation process. The volume of the spheres ranged from 1 to $15 \mathrm{ml}$. The CT scan was acquired in a spiral mode, with an axial motion of $3 \mathrm{~mm}$ per scanner rotation and the images were reconstructed with a $3.4 \mathrm{~mm}$ axial spacing to match that of the PET images. The PET scan, with a deadtime of 5\% and random coincidence fraction of $20 \%$, collected $\sim 10^{7}$ coincidences to match a typical 10 min clinical scan.

Precise alignment of the PET and CT images was obtained with two calibration procedures. In the first procedure an array of parallel 18-gauge steel needles $(<0.5 \mathrm{~mm}$ diameter $)$ filled with aqueous $[18 \mathrm{~F}]$ solution was scanned in both CT and PET mode. From the reconstructed images a precise determination of the pixel size of each modality was made. The residual translation and rotation offsets in the transverse plane between modalities was also measured. For the second procedure, an array of four-point sources (each $\sim 1.5 \mathrm{~mm}$ diameter) was attached to the top of the torso phantom by means of glass capillary tubes $(\sim 0.5 \mathrm{~mm}$ diameter $)$. The point sources were scanned in CT mode with a $1 \mathrm{~mm}$ axial spacing. From the reconstructed PET and CT images the precise axial offset and out-of-plane rotation of the torso phantom was measured.

Finally the CT image of the torso phantom was re-sampled to correct for the residual PET-CT alignment errors, converted to voxel labels as described above and incorporated in the PET image reconstruction according to equations (1)-(3).

\section{Results}

\subsection{Simulation studies}

4.1.1. Noiseless 2D data. Figure 2 shows the noiseless 2D test object and the horizontal profiles through the reconstructed images for the five different combinations of labels and reconstruction methods described above in section 3.1.1. Figure 2(i) shows a typical loss of 


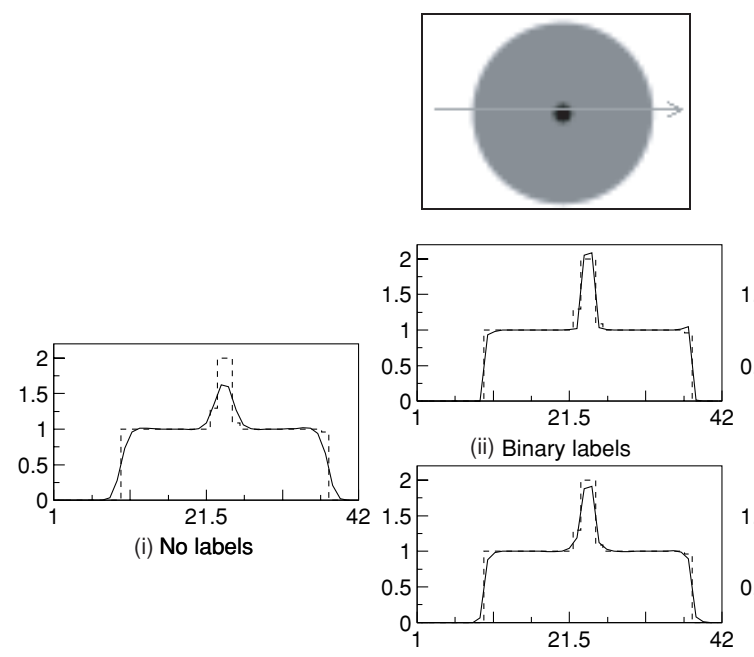

(iii) Blurred labels

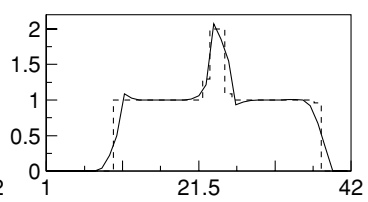

(iv) Shifted binary labels

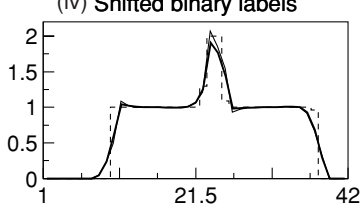

(v) Shifted blurred labels

Figure 2. The noiseless test object and horizontal profiles through the reconstructed PET emission images using PWLS with five different combinations of labels and penalty terms: (i) no labels, (ii) correct labels with binary weights, (iii) correct labels with blurred weights, (iv) shifted labels with binary weights, and (v) shifted labels with blurred weights. For the later graph (v), to the blurred weights profile drawn in bold, the binary weights profile (iv) is superposed. All five PWLS reconstructions were performed with the same image smoothness parameter $\beta$. For comparison, the horizontal profile through the original (simulated) object is shown with a dashed line in each graph.

resolution due to regularization, while the inclusion of binary labels in figure 2(ii) allows for near-perfect contrast recovery. The use of blurred label maps (figure 2(iii)) results in a small loss in contrast recovery, as expected. When mispositioned labels are used, the reconstructed image is distorted (figure 2(iv)), which is marginally mitigated by the use of blurred labels (figure 2(v), indicated by the bold profile with reduced over- and undershoot around pixel 12 and 26).

4.1.2. Noisy 3D data. Two of the 100 realizations are shown in figure 3 for the three cases of: no labels, binary labels and blurred labels (FWHM of $5 \mathrm{~mm}$ ). The corresponding mean and standard deviation images are shown in figure 4 for each of the three label combinations. For comparison, the individual realizations and the mean images shown below are scaled to the same maximum and minimum display range as the original object shown in figure 1(a). Figure 4 illustrates increased contrast recovery when binary labels are used. The noise also increases, particularly around the edges of the contrast objects, as expected for an edgepreserving prior. The use of blurred labels results in contrast recovery and noise levels that lie between the cases of no labels and binary labels. The measured noise levels are shown in figure 5, which plots the standard deviation, $s$, (equation (7)), relative to the no labels case, as a function of the object size. The relative standard deviations are given for two representative amounts of smoothing, as controlled by the regularization parameter $\beta$ in equation (1).

The results from simulating the case where there is a $5 \mathrm{~mm}$ positioning error are shown in figures 6 and 7 for the same image smoothness parameter. Figure 6 shows the mean and standard deviation images for reconstructions with binary labels and blurred labels (FWHM 
Realization A:
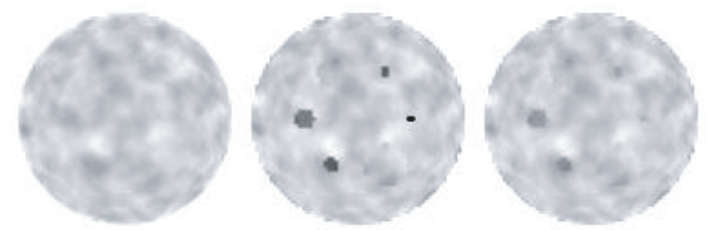

Realization B:

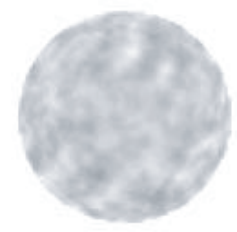

No labels

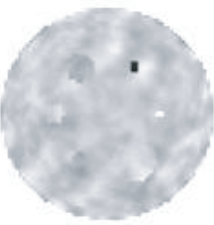

Binary labels

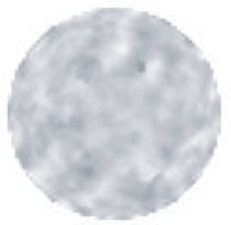

Blurred labels

Figure 3. Reconstructions of two representative realizations of the 100 simulations with accurate labels. The top and bottom rows are the two independent realizations, while the columns are, from left to right, images reconstructed with: no labels, binary labels and blurred labels.

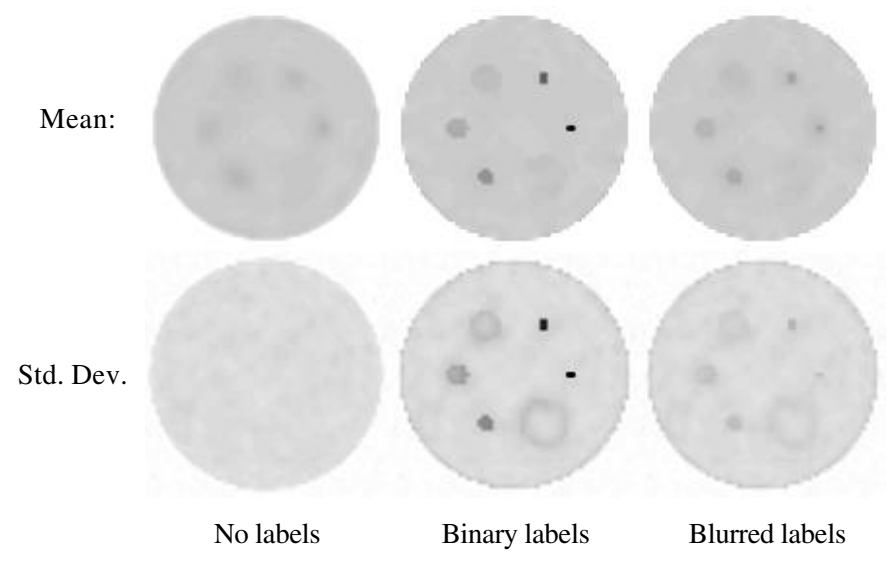

Figure 4. Mean and standard deviation images of the 100 realizations of simulations with accurate labels. Top row: mean images. Bottom row: standard deviation images. The columns correspond to figure 3 and are, from left to right: no labels, binary labels and blurred labels.

of $5 \mathrm{~mm}$ ). Images reconstructed without labels are identical to those shown in figure 4. Also indicated is the location of the profiles plotted in figure 7 for the original and shifted labels. Figure 7 shows that when binary labels are used, mis-positioning of the label essentially results in mis-positioning of the corresponding objects in the emission image, similar to figure 2(iv). The use of blurred labels mitigates this distortion, but at the cost of reduced contrast recovery.

The bias (equation (4)) versus standard deviation (equation (7)) curves were plotted for all combinations of label type and image smoothness parameter $\beta$, for both accurate and shifted labels. The results for all the objects varied gradually according to the size of the object and $\beta$, so only representative results are shown in figure 8 for the 15 and $40 \mathrm{~mm}$ diameter objects, shown at the top of figure 1(a). These plots show that the use of accurate labels improves noise/contrast trade-off, with the amount of improvement increasing with object diameter. The use of mispositioned labels, however, yields results that are equivalent to not using labels 


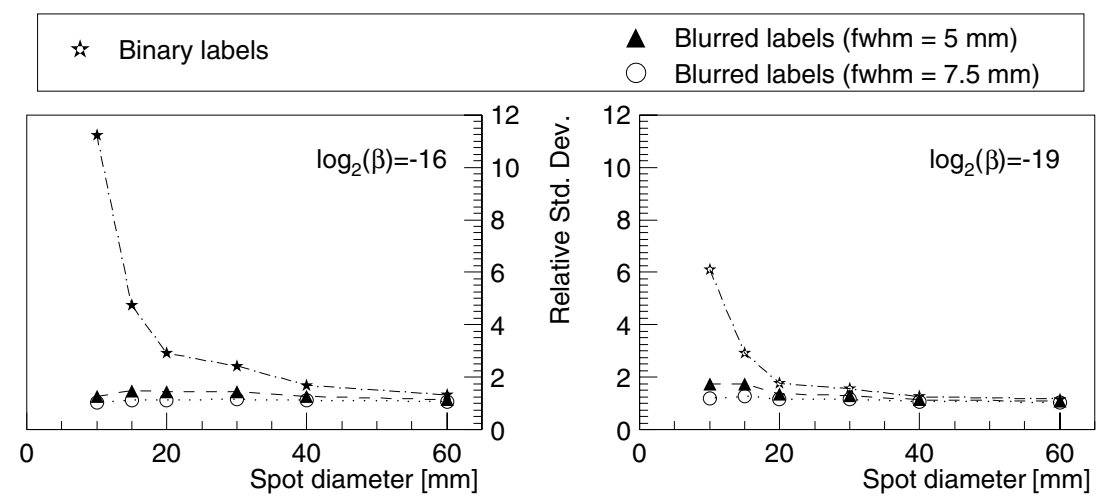

Figure 5. Ensemble standard deviation of ROI mean values relative to that for the reconstruction without labels. These are shown as a function of the diameter of the six labels for two different levels of regularization. The left plot, with $\beta=2^{-16}$ in equation (1), corresponds to increased regularization or image smoothness compared to the right plot, where $\beta=2^{-19}$.

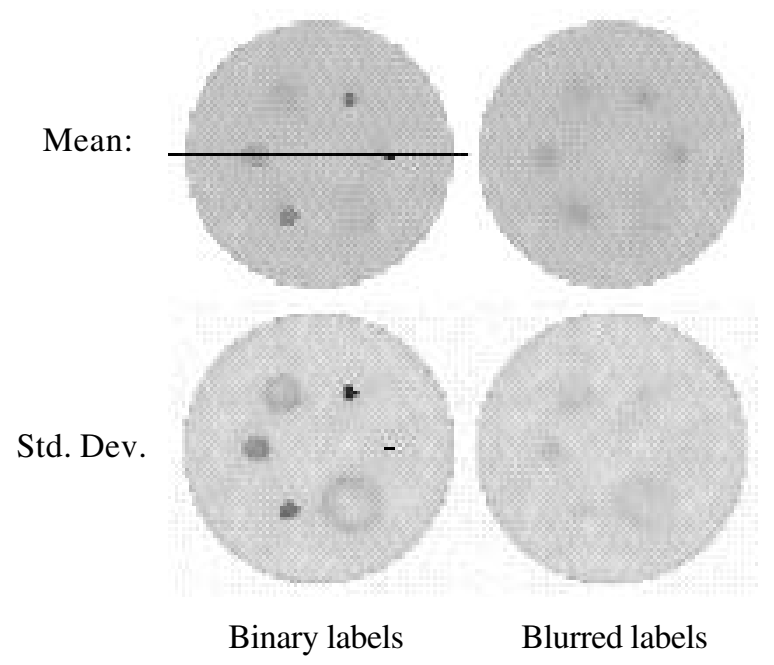

Figure 6. Mean and standard deviation images of the 100 reconstructed images with shifted labels. Top row: mean images. Bottom row: standard deviation images. The columns are, left: binary labels, right: blurred labels. Also indicated is the location of the profiles plotted in figure 7.

at all. Figure 8 also indicates the complex relation between the width of the kernel used to blur the labels and the regularization parameter, $\beta$. As either $\beta$ or the kernel width increase, the noise is reduced and the bias increases.

The RMS error (equation (10)) curves were calculated over a wide range of the image smoothness parameter $\beta$ to see the asymptotic RMS values corresponding to the case of no penalization $\left(\log _{2}(\beta)<-30\right)$ and to the case of almost 'full' penalization $\left(\log _{2}(\beta)>-6\right)$. Typical values that match the range of cutoff frequencies used with filtered-backprojection in clinical imaging are $-20 \leqslant \log _{2}(\beta) \leqslant-14$ (Comtat et al 1998). The results are shown in figure 9 for the three smallest objects (diameters from 10 to $20 \mathrm{~mm}$ ). As expected, when $\beta$ tends to zero $\left(\log _{2}(\beta)<-30\right)$, the regularization has almost no effect and the RMS errors are the same regardless of the type of penalty weight. At the other extreme $\left(\log _{2}(\beta)>-6\right)$, 

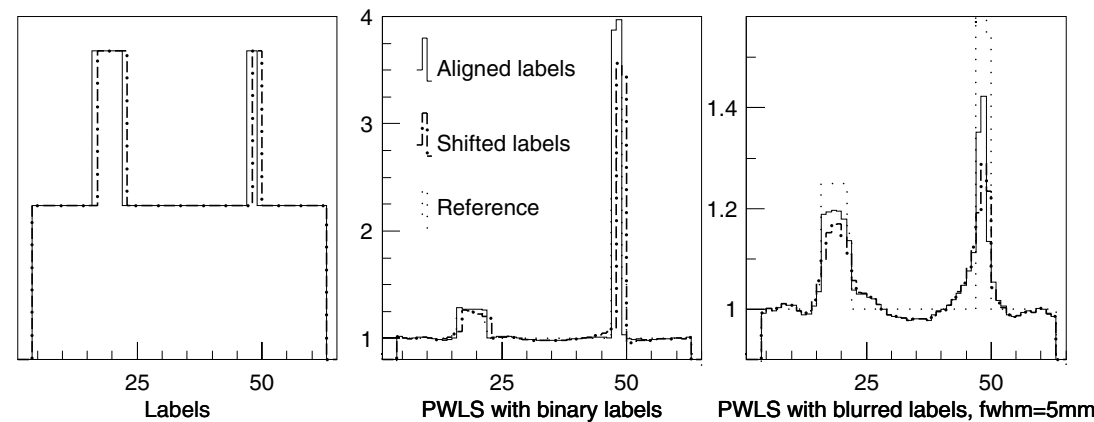

Figure 7. Line profiles corresponding to figure 6 showing the effect of shifted labels. Left: Original (true) and $5 \mathrm{~mm}$ positional shift of labels. Effect on images reconstructed using labels: binary labels (centre), blurred labels (right). Note the change in vertical scales and that the horizontal axis is in units of reconstructed image pixels. The true image profile is also shown for reference.

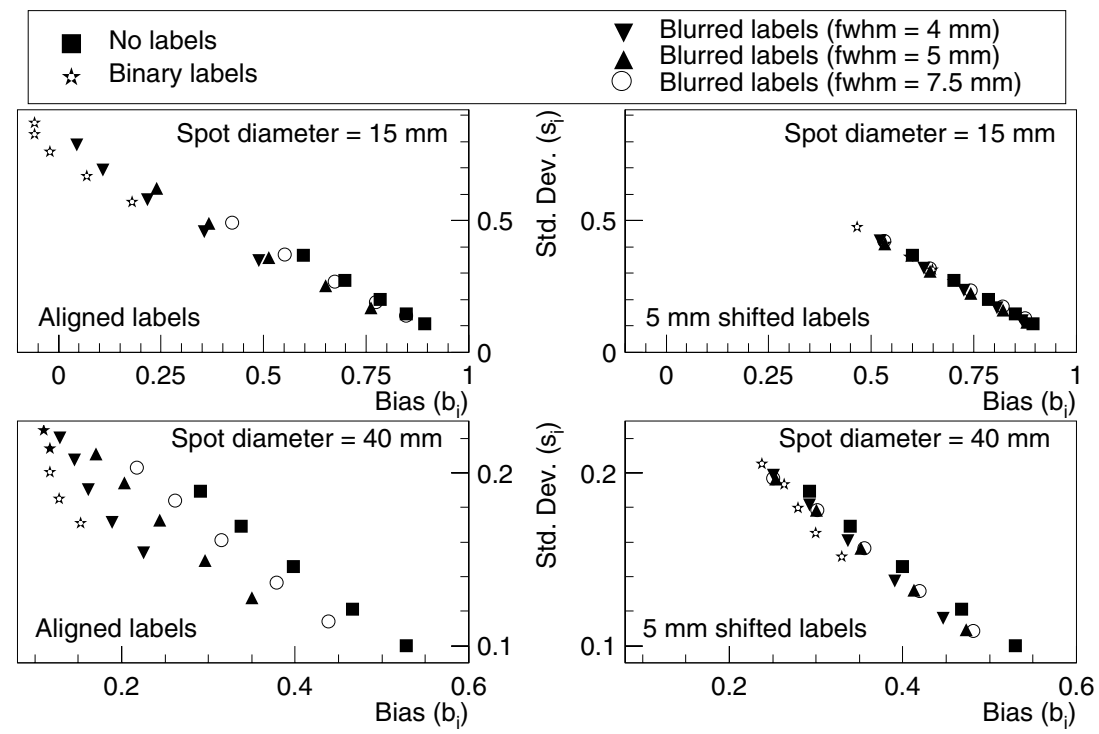

Figure 8. Standard deviation $\left(s_{i}\right)$ versus bias $\left(b_{i}\right)$ graphs for both accurate and shifted labels for the top two contrast objects in figure 1(a) as a function of the regularization parameter and type of penalty weight.

for aligned labels, the use of binary labels results in a lower asymptotic RMS value. For the $5 \mathrm{~mm}$ shifted labels, for the same image smoothness parameter $\beta$, the RMS error is increased relative to the use of aligned labels.

For typical values of the image smoothness parameter $\beta\left(-20 \leqslant \log _{2}(\beta) \leqslant-14\right)$, the use of binary labels, even perfectly aligned, results in some RMS values higher than those without using labels. Table 1 summarizes the RMS error values for the image smoothness parameter $\beta$ that minimizes the RMS value without labels ( $\mathrm{RMS}_{\mathrm{MIN}}$ ). For the $5 \mathrm{~mm}$ shifted labels, the RMS values for the binary labels are always higher than $\mathrm{RMS}_{\mathrm{MIN}}$, while the RMS values for the blurred labels are slightly lower.

The effect of false labels is demonstrated in the two realizations shown in figure 10 and the mean and standard deviation images shown in figure 11. The relative standard deviations 


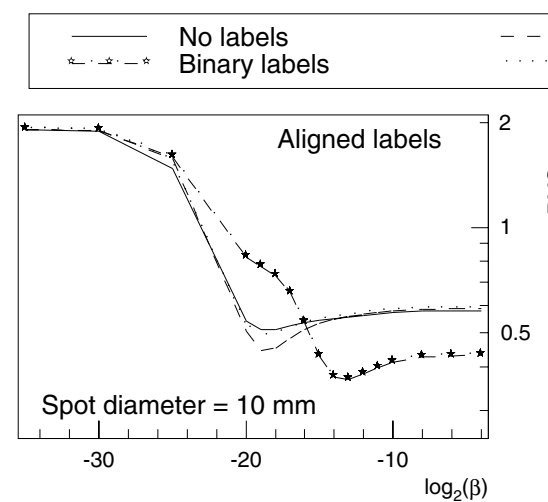

Blurred labels (fwhm $=5 \mathrm{~mm}$ ) Blurred labels (fwhm $=7.5 \mathrm{~mm}$ )
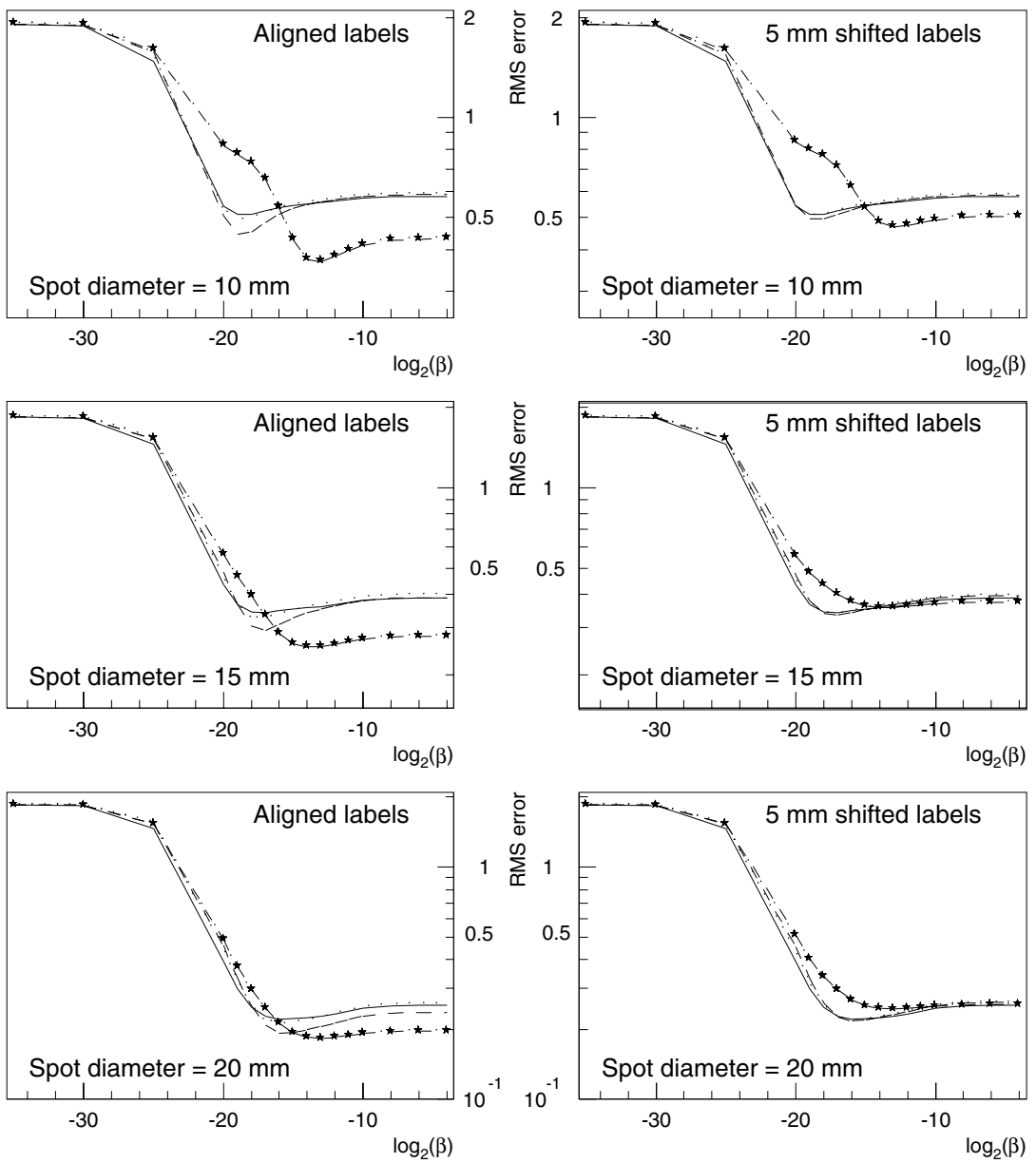

Figure 9. RMS error versus image smoothness parameter $\beta$ for the three smallest objects in figure 1(a), for both accurate (left) and shifted (right) labels.

for the case of false labels were essentially identical to those shown in figure 5, indicating that while false labels do not add bias, they substantially increase variance for the regions associated with the false labels. The effect of false labels could thus lead to false-positives for the detection task, as indicated by figure 10. It is interesting to note the similarity between the standard deviation images in figures 6 (true labels) and 11 (false labels) while the corresponding mean images show the lack of bias in both cases.

\subsection{Experimental studies}

The needle sources measured in PET and CT showed that the voxel dimensions differed by $1.8 \%$ throughout the field of view. There was a residual in-plane rotational offset of $0.5^{\circ}$ but no residual transverse shift or out-of-plane rotation between modalities. By inspection of the overlaid PET and CT images of the needle and point source arrays, the final image alignment 
Table 1. RMS error values for the three smallest object in figure 1(a). The image smoothness parameter $\beta$ that minimizes the RMS in absence of labels ("no label' column) was selected for each object. For this parameter, the RMS is reported for three different types of labels, with both accurate and shifted labels.

\begin{tabular}{|c|c|c|c|c|c|c|c|c|}
\hline \multirow{3}{*}{$\begin{array}{l}\text { Spot diameter } \\
(\mathrm{mm})\end{array}$} & \multirow[b]{3}{*}{$\log _{2}(\beta)$} & \multicolumn{7}{|c|}{ RMS error } \\
\hline & & \multirow[b]{2}{*}{ No labels } & \multicolumn{2}{|c|}{ Binary labels } & \multicolumn{2}{|c|}{$\begin{array}{c}\text { Blurred labels } \\
\text { FWHM = } 5 \mathrm{~mm}\end{array}$} & \multicolumn{2}{|c|}{$\begin{array}{c}\text { Blurred labels } \\
\text { FWHM }=7.5 \mathrm{~mm}\end{array}$} \\
\hline & & & Aligned & $5 \mathrm{~mm}$ shift & Aligned & $5 \mathrm{~mm}$ shift & Aligned & $5 \mathrm{~mm}$ shift \\
\hline 10 & -19 & 0.511 & 0.775 & 0.799 & 0.446 & 0.497 & 0.490 & 0.503 \\
\hline 15 & -17 & 0.342 & 0.333 & 0.400 & 0.294 & 0.334 & 0.324 & 0.336 \\
\hline 20 & -16 & 0.222 & 0.213 & 0.268 & 0.193 & 0.217 & 0.211 & 0.219 \\
\hline
\end{tabular}

Realization C:
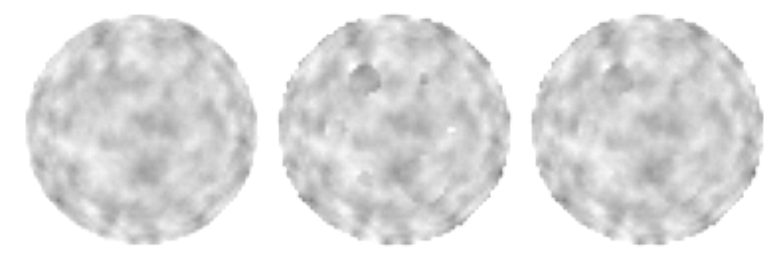

Realization D:

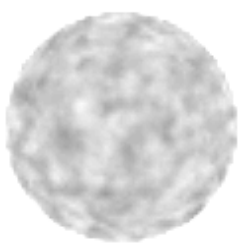

No labels

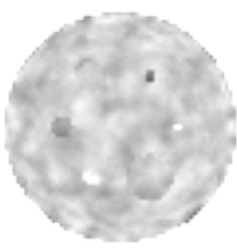

Binary labels

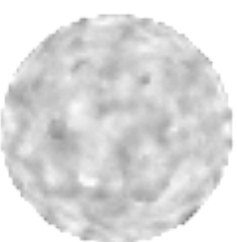

Blurred labels

Figure 10. Effect of false labels. Two realizations from simulations with false labels and a uniform emission distribution. The top and bottom rows are independent realizations, while the columns are, from left to right: no labels, binary labels and blurred labels.

Mean:
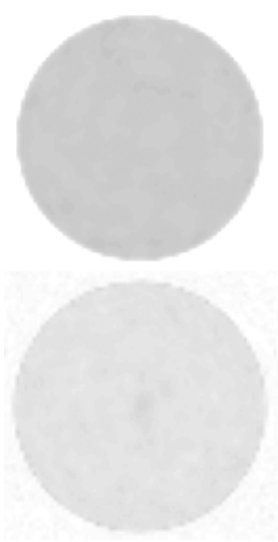

No labels
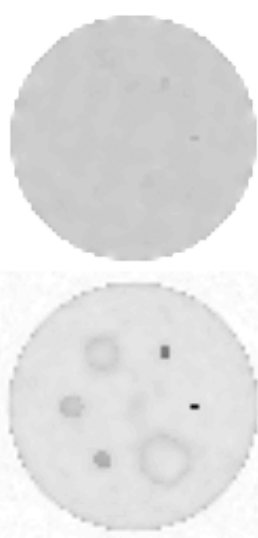

Binary labels
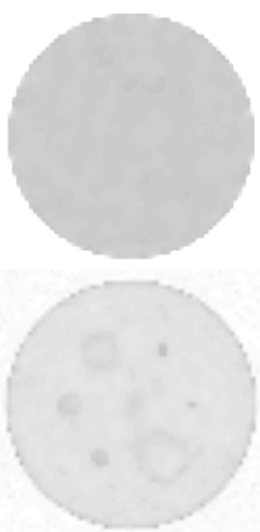

Blurred labels

Figure 11. Mean and standard deviation images of the 100 realizations of simulations with false labels. Top row: mean images. Bottom row: standard deviation images. The columns correspond to figure 10 and are, from left to right: no labels, binary labels and blurred labels. 


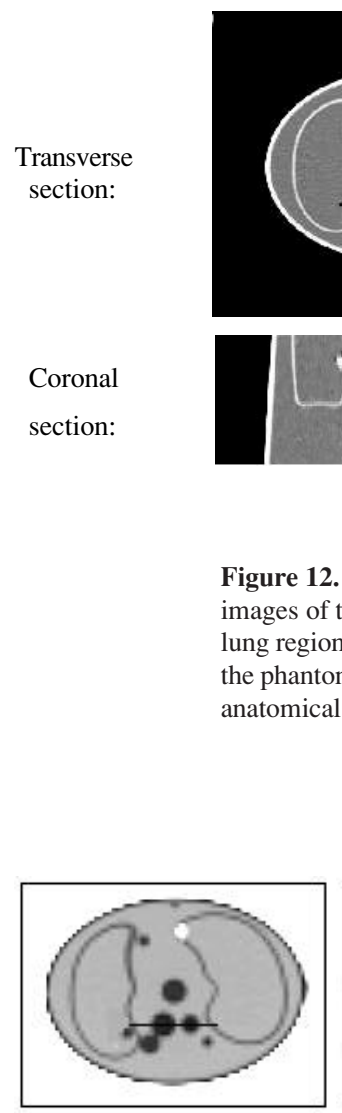

Profile location

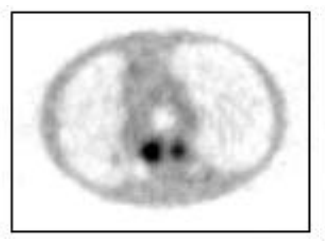

No labels

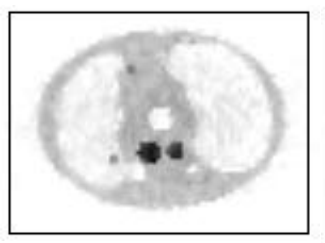

Binary labels
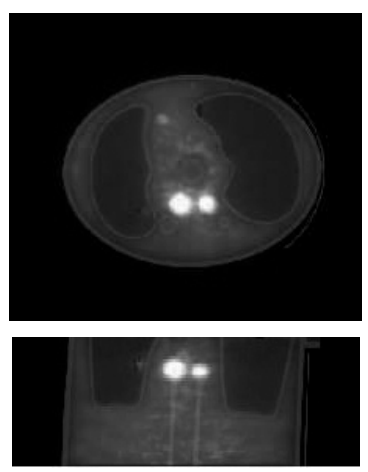

Fused PET/CT
PET (no labels)

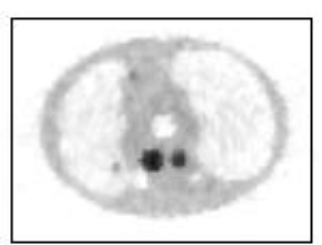

Blurred labels

Figure 13. Transverse sections through reconstructed volume images of the measured 3D phantom (with $\beta=2^{-15}$ ) showing four hot contrast objects and three cold contrast objects.

accuracy after correcting for the voxel-scaling difference and residual rotation offset was estimated to be $<2 \mathrm{~mm}$ throughout the field of view. Figure 12 shows the transverse and frontal sections through the reconstructed CT and PET volume images and the fused PET/CT volume image.

Transverse sections through the reconstructed volume PET images are shown in figure 13. Representative images are shown for penalty weights based on the three cases of no labels, binary labels, and blurred labels. The image reconstructed with binary labels clearly demonstrates improved contrast for the smaller hot and cold spheres, while the use of the blurred labels results in contrast levels in between those obtained with and without binary labels. Profiles through the reconstructed images are displayed in figure 14, demonstrating the improved contrast with the use of binary and blurred labels.

The processing time for the FORE-rebinning, the PWLS and PWLS + labels reconstructions given separately in table 2. For reference, the reconstruction time using 2D filtered-backprojection (FBP) is also given. Comparisons of images reconstructed (without anatomical a priori information), by FORE + FBP, and the combinations of FORE + PWLS, FORE + OSEM, and FORE + AWOSEM, were discussed by Comtat et al (1998). 


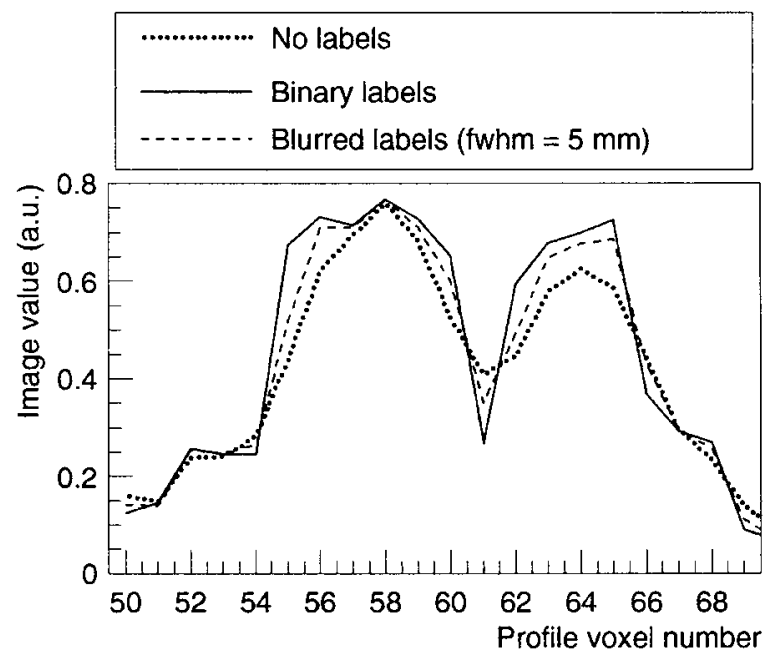

Figure 14. Profiles through the images of figure 13 showing the effect of including anatomical information with measured data. Solid line: no labels, dotted line: binary labels, dashed line: blurred labels.

Table 2. Computation time of the FORE + PWLS reconstruction method on a Sun Blade 1000 workstation, the for one-bed position of the PET/CT scanner (47 image planes). For purposes of comparison, we also reported the computation time of 2D-FBP (Comtat et al 1998).

\begin{tabular}{lc}
\hline Technique & Computation time (s) \\
\hline FORE & 80 \\
FBP 2D & 30 \\
PWLS, 30 iterations, no labels & 630 \\
PWLS, 30 iterations, binary labels & 660 \\
PWLS, 30 iterations, blurred labels & 810 \\
\hline
\end{tabular}

\section{Discussion and conclusions}

As mentioned in section 1, our goal was not necessarily to develop a method that includes anatomical a priori information more accurately than existing methods (Leahy and Yan 1991, Gindi et al 1993, Bowsher et al 1996), but rather to develop a method that would (i) reconstruct 3D multi-bed data in clinically feasible time (achieved with the FORE and SOR algorithms, as shown in table 2), (ii) include a reasonably accurate model of the positron imaging statistics (achieved by estimating the data variance) (iii) converge (achieved by using fixed weights in the penalty function), and (iv) introduce few free parameters (achieved by only allowing the width of the blurring kernel as a free parameter).

To utilize anatomical (CT) information in the functional (PET) image reconstruction we presented a 3D voxel label model that can accommodate any 3D voxel labeling topology. This information is incorporated anatomical information via the penalty weights of the PWLS objective function. This combination of FORE + PWLS + labels was developed as it allows for both reconstruction of 3D whole-body datasets in clinically feasible times and also inclusion of anatomical information in such a way that convergence can be guaranteed.

To reduce the deleterious effects of mismatches between the PET and CT data, the anatomical labels derived from the $\mathrm{CT}$ data are blurred by a pre-specified amount corresponding 
to the alignment accuracy. The blurred label information is not updated during the PET image reconstruction. An alternative approach to that taken here is to estimate the anatomical boundaries jointly with emission distribution, with the CT or MRI image used to 'guide' estimated positions of functional boundaries (Leahy and Yan 1991, Gindi et al 1993, Bowsher et al 1996). Joint estimation can potentially compensate for alignment errors (Zhou et al 1993), and can be incorporated into the blurred label approach. This could be done by, for example, re-estimating the label maps at pre-determined iterations of the PET image reconstruction using both the CT image and the current PET image. With the joint estimation methods mentioned above, Bayesian modelling of the prior probability of the anatomical and functional boundaries can allow for more accurate alignment. These techniques can thus potentially circumvent the problems introduced by anatomical and functional mismatches (as demonstrated by Zhou et al 1993), albeit at the cost of increased computation time. In addition, the use of variable weights $\omega_{i k}$ can lead to a non-convex penalty function and so global convergence would no longer be assured unless more sophisticated estimation methods are used. Keeping the anatomical information fixed, however, while estimating the PET image leads to a simpler and faster procedure with fewer free parameters to be pre-specified, a consideration important for the methods intended for routine clinical use, particularly with 3D whole-body PET scanning. But this simplicity has a price: to allow for mismatches, the overall quality of the anatomical information is degraded by the use of fixed blurred labels, limiting the maximum achievable accuracy in the PET image that can be obtained, while Bayesian joint-estimation methods can potentially recover from mismatches and thus retain, at least locally, the original quality of the anatomical information. If rapidity and simplicity are not a critical issue, or with sufficient processing speed, the use of Bayesian joint-estimation methods could utilize the full accuracy of the anatomical information in the PET image reconstruction, even if (i) there is an alignment error between the CT and PET data (positional mismatch) or (ii) if there is a true object boundary present in the PET data that is not present in the CT data (signal mismatch). The latter situation can arise in oncology imaging for tumours with heterogeneous uptake and uniform tissue density. It is thus possible that both estimation and detection tasks could be improved with a carefully chosen Bayesian joint-estimation method, relative to the use of the blurred labels method described here. However, the reliability of Bayesian joint-estimation methods with the low count levels typical of clinical 3D whole-body PET imaging has not been investigated, as most implementations have been tested with the significantly higher count levels encountered in PET brain imaging studies. In our investigation we did not compare the blurred label method with fully 3D implementations of the alternative approaches of including anatomical a priori information mentioned above, but rather relied on our comparisons with the standard PWLS method to demonstrate the efficacy of the blurred label approach for clinical 3D whole-body PET imaging.

Some of the potential advantages and problems of including anatomical information are apparent in figure 2. With accurate alignment, near-perfect contrast recovery is obtained for these simple (piecewise-constant) objects. With the use of binary labels, however, a positional mismatch distorts the reconstructed object as shown by figures 2 and 7. Label maps blurred with a one-pixel Gaussian kernel (FWHM) reduce edge artefacts while also retaining a better contrast than the PWLS reconstruction without anatomical information.

For the simulations with statistical noise the improvements in the noise-bias trade-offs were more apparent for the larger objects (figure 8) even though they had a lower contrast and varied with the type of anatomical label used. In general, the use of binary labels resulted in a dramatic increase in variance, which was reduced by blurring the label maps (figures 4 , 5 and 8). Even with accurate labels, the RMS error can be increased by the use of binary labels (figure 9 and table 1) for values of $\beta$ that match the range of cut-off frequencies used 
with filtered-backprojection in clinical imaging (Comtat et al 1998). The RMS error figure of merit accounts not only for bias in the image, but also for noise. With binary labels, image variance also increases in the neighbourhood of the label boundaries (figure 4); this effect counterbalances the decreased bias.

Although we are interested in improving the estimation by including anatomical a priori information, we can also consider the effects on detection. One potential pitfall of including anatomical information is the temptation to over-smooth images to improve the contrast. In the presence of signal mismatches this may lead to increased false-positive detection rates (due to false labels) and/or false-negative detection rates (due to missing labels). This is anecdotally illustrated in figure 3 for missing labels (the 'no labels' case) and figure 10 for false labels. These effects are more dramatic for the false labels case when binary labels are used, rather than blurred labels, as shown by the standard deviation images in figure 11 . The use of blurred labels reduced, but did not eliminate the variance in the regions of the false labels. Not surprisingly, the standard deviation images in the cases of accurate and false labels were very similar. The appearance of false-positive and/or false-negative objects (figures 3 and 10) are specific to the parameters of the study. In the case of a positional mismatch it is difficult to make any conclusions on the effect of labels for the detection task. The plots in figures 8 and 9 show that the use of accurate labels improves noise/contrast trade-offs and reduces RMS error, while mis-positioned labels yield results that are roughly equivalent to not using labels at all. The reasons for this are not clear, but it may be that mis-positioning the labels is somehow equivalent to over-smoothing the images. The use of labels, however, in the case of a positioning mismatch, does not lead to an increase in bias relative to not using labels; an important consideration for quantitation tasks.

Even with observer ROC studies it is not obvious what task performance should be measured. For example, in the case of a positional mismatch where detection is improved at the expense of a localization error, it is not clear if this should be considered an improvement or not as the PET image (reconstructed with labels) could be used for lesion detection, while the CT image is used for localization. Other variations are possible and these questions cannot be answered until considerably more experience is gained with combined PET/CT studies.

For the estimation task, in the case where the labels were shifted by $5 \mathrm{~mm}$ (one pixel) from the true emission contrast regions, there was a reduction in image contrast, as shown by comparing the mean images in figures 4 and 6 . In addition, there was a shift in the apparent centroid of the objects that corresponded to the shift in the label locations, as shown in figure 7. There was also a significant reduction in ROI variance. The net effect is shown in figure 8 for two of the objects, indicating that a $5 \mathrm{~mm}$ shift is sufficient to remove any advantage of using anatomical information in terms of contrast/noise trade-off. We note, however, that this amount of label misalignment does not degrade the noise-bias performance relative to PWLS without anatomical information.

The experimental studies, which had an alignment accuracy of better than $2 \mathrm{~mm}$, indicate that the use of both binary and blurred labels leads to improved contrast for the hot and cold contrast objects of all sizes, as illustrated in figure 13. It should be noted, however, that both the experimental and simulated phantoms are piece-wise continuous objects and are ideally suited for this type of reconstruction method. It may be, in practice, that methods favouring piece-wise-linear objects, such as those proposed by Lee et al (1995), provide more accurate reconstructions. It should be emphasized that the blurred labels method is not a substitute for the Bayesian joint functional/anatomical estimation methods proposed in the literature, but rather a simpler and faster, but potentially less precise, alternative for clinically feasible 3D whole-body PET imaging with a PET/CT scanner. 
In summary, PET/CT tomographs, by directly acquiring aligned PET and CT data, offer the possibility of using anatomical information to guide the reconstruction of whole-body PET data. With mismatches between the PET and CT data, the effects on detection or estimation tasks should be considered separately. For detection tasks, signal mismatches will likely increase the false-positive and/or false-negative detection rates, depending on the type of mismatch, although the increased variance introduced by the false labels can be reduced by the use of blurred labels. The effect of positional mismatches is difficult to evaluate in a realistic manner. For estimation procedures with either positional or signal mismatches, the use of blurred labels appears to lead to a performance no worse than that of images reconstructed without anatomical information. With accurate PET-CT alignment, the use of anatomical labels improves the RMS error and bias-variance trade-off relative to images reconstructed without anatomical information. In this case the performance for both detection and estimation tasks will likely be improved.

\section{Acknowledgments}

This work is supported by a grant from the Swiss National Science Foundation and NIH grants CA74135 and CA65856.

\section{References}

Ardekani B A, Braun M, Hutton B F, Kanno I and Iida H 1996 Minimum cross-entropy reconstruction of PET images using prior anatomical information Phys. Med. Biol. 41 2497-517

Beyer T, Townsend D W, Brun T, Kinahan P E, Charron M, Roddy R, Israel J, Jerin J, Young J, Byars L and Nutt R 2000 A combined PET/CT scanner for clinical oncology J. Nucl. Med. 41 1369-79

Bowsher J E, Johnson V E, Turkington T G, Jaszczak R J, Floyd C E, Jr and Coleman R E 1996 Bayesian reconstruction and use of anatomical a priori information for emission tomography IEEE Trans. Med. Imaging 15 673-86

Comtat C, Kinahan P E, Defrise M, Michel C and Townsend D W 1998 Fast reconstruction of 3D PET data with accurate statistical modeling IEEE Trans. Nucl. Sci. 45 1083-9

Comtat C, Kinahan P E, Defrise M, Michel C and Townsend D W 1999 Simulating whole-body PET scanning with rapid analytical methods 1999 IEEE Nuclear Science Symposium and Medical Imaging Conference (Seattle, WA) pp 1260-4

Defrise M, Kinahan P E, Townsend D W, Michel C, Sibomana M and Newport D F 1997 Exact and approximate rebinning algorithms for 3-D Pet data IEEE Trans. Med. Imaging 16 145-58

Fessler J A 1994 Penalized weighted least squares image reconstruction for positron emission tomography IEEE Trans. Med. Imaging 13 290-300

Fessler J A, Clinthorne N H and Rogers W L 1992 Regularized emission image reconstruction using imperfect side information IEEE Trans. Nucl. Sci. 39 1464-71

Furuie S S, Herman G T, Narayan T K, Kinahan P E, Karp J S, Lewitt R M and Matej S 1994 A methodology for testing for statistically significant differences between fully 3D PET reconstruction algorithms Phys. Med. Biol. 39 341-54

Geman S and Geman D 1984 Stochastic relaxation, Gibbs distributions, and the Bayesian restoration of images IEEE Trans. Pattern Anal. Mach. Intel. 6 721-41

Gindi G, Lee M, Rangarajan A and Zubal G 1993 Bayesian reconstruction of functional images using anatomical information as priors IEEE Trans. Med. Imaging 12 670-80

Hudson H and Larkin R 1994 Accelerated image reconstruction using ordered subsets of projection data IEEE Trans. Med. Imaging 13 601-9

Hurn M, Mardia K, Hainsworth T, Kirkbride J and E B 1996 Bayesian fused classification of medical images IEEE Trans. Med. Imaging 15 850-8

Johnson V E, Bowsher J E, Jaszczak R J and Turkington T G 1995 Analysis and Reconstruction of Medical Images Using Prior Information Case Studies in Bayesian Statistics vol II ed G Gatsonis, J S Hodges, R E Kass and N D Singpurwalla (New York: Springer) pp 149-218

Karssemeijer N 1990 A statistical method for automatic labeling of tissues in medical images Machine Vision and Applications $375-86$ 
Kinahan P E, Michel C, Defrise M, Townsend D W, Sibomana M, Lonneux M, Comtat C and Luketich J D 1997 Accelerated statistical reconstruction methods for PET and coincidence-SPECT wholebody oncology imaging J. Nucl. Med. 38102

Kinahan P E, Townsend D W, Beyer T and Sashin D 1998 Attenuation correction for a combined 3D PET/CT scanner Med. Phys. 25 2046-53

Leahy R and Qi J 2000 Statistical approaches in quantitative positron emission tomography Stat. Comput. 10 147-65

Leahy R and Yan X 1991 Incorporation of anatomical MR data for improved functional imaging with PET Information Processing in Medical Imaging: 12th International Conference (Wye, UK) (Berlin: Springer) pp 105-120

Lee S-J, Rangarajan A and Gindi G 1995 Bayesian image reconstruction in SPECT using higher order mechanical models as priors IEEE Trans. Med. Imaging. 14 669-80

Liu X, Comtat C, Michel C, Kinahan P E, Defrise M and Townsend D W 2001 Comparison of 3D reconstruction with 3D-OSEM and with FORE + OSEM for PET IEEE Trans. Med. Imaging 20 804-14

Mumcuoglu E, Leahy R, Cherry S and Zhou Z 1994 Fast Gradient-based methods for Bayesian reconstruction of transmission and emission PET images IEEE Trans. Med. Imaging 13 687-701

Ollinger J M and Fessler J A 1997 Positron emission tomography IEEE Signal Process. Mag. 14 43-55

Ouyang X, Wong W H, Johnson V E, Hu X and Chen C T 1994 Incorporation of correlated structural images in PET image reconstruction. IEEE Trans. Med. Imaging 13 627-41

Rigo P, Paulis P, Kaschten B J, Hustinx R, Bury T, Jerusalem G, Benoit T and Foidart-Williams J 1996 Oncological applications of positron emission tomography with fluorine-18 fluorodeoxyglucose Eur. J. Nucl. Med. 23 1641-74

Rowe R W and Dai S 1992 A pseudo-Poisson noise model for simulation of positron emission tomographic projection data Med. Phys. 19 1113-9

Sauer K and Bouman C 1993 A local update strategy for iterative reconstruction from projections IEEE Trans. Signal Process. 41 534-48

Weber W A, Avril N and Schwaiger M 1999 Relevance of positron emission tomography (PET) in oncology Strahlentherapie und Onkologie 175 356-73

Zhou Z, Leahy R M and Mumcuoglu E U 1993 A comparative study of the effects of using anatomical priors in PET reconstruction The 1993 IEEE Nucl. Sci. Symp. Med. Imaging Conf. pp 1749-53 